\title{
Myocellular characteristics in rheumatoid arthritis and osteoarthritis patients
}

\author{
Sandro Manuel Mueller ${ }^{1,2}$, David Aguayo ${ }^{2,6}$, Daniel Aeberli ${ }^{3}$, Esther Vögelin ${ }^{4}$ and Marco Toigo ${ }^{2,5^{*}}$
}

Duijnisveld et al. have published an interesting study on the regenerative potential of muscle satellite cells in chronic inflammation in this journal [1]. They showed that muscle stem cell populations obtained from $M$. vastus medialis of patients with rheumatoid arthritis (RA) and osteoarthritis (OA) exhibited similar myogenic purity, viability, growth speed, differentiation, and maximum proliferative capacity. Based on these findings in vitro, the authors hypothesized that circulating inflammatory factors in RA negatively influence the regenerative potential of satellite cells and muscle strength in vivo. We aimed to verify whether these results obtained from vastus medialis muscles also apply to a muscle typically involved in the disease process of RA, namely $M$. interosseus dorsalis manus 1.

For this purpose, we obtained intraoperative muscle biopsies from the $M$. interosseus dorsalis manus 1 of five RA (57.2 \pm 11.1 years old) and four OA (60.7 \pm 12.1 years old) patients and tested whether satellite cell numbers, myofiber sizes, and proportions were different between RA and OA patients. There was no difference in muscle fiber type distribution between RA and OA patients (Table 1). Myofiber cross-sectional area (CSA), myonuclear domains, the number of $\operatorname{Pax} 7^{+}$cells, and the number of proinflammatory macrophages $\left(\mathrm{CD}^{+} 8^{+}\right)$were not different between RA and OA patients. There was a tendency for increased myonuclear number in myosin heavy chain (MyHC)-1 fibers in RA patients compared with OA patients, while there was no difference in myonuclear number in MyHC-2 fibers between the groups. MyHC-2 fiber CSAs in M. interosseus dorsalis manus 1 were significantly larger than MyHC-1 CSAs in RA and OA patients (Table 1).

Our results point towards similar muscle characteristics between RA and OA patients in the highly

\footnotetext{
* Correspondence: marco.toigo@oym.ch

${ }^{2}$ Institute of Human Movement Sciences, ETH Zurich, Zurich, Switzerland

${ }^{5}$ Research and Performance Centre for Elite Athleticism, OYM,

Lorzenparkstrasse 12, 6330 Cham, Switzerland

Full list of author information is available at the end of the article
}

affected M. interosseus dorsalis manus 1. Moreover, we found that most values for RA patients seemed to be higher when compared with $\mathrm{OA}$ in this preliminary dataset. Notably, there was a tendency for increased myonuclear number in $\mathrm{MyHC}-1$ fibers in $\mathrm{RA}$ patients. Our results from a severely affected skeletal area are in line with previous studies investigating other skeletal sites. In M. vastus medialis, MyHC-2 CSAs were significantly larger than MyHC-1 CSAs in RA patients [2] and no significant differences in satellite cell numbers between RA and OA patients were present [3]. Based on our results from a small patient sample, the hypothesis that chronic systematic inflammation negatively influences the regenerative potential of satellite cells and myonuclei number cannot be confirmed, but it warrants further investigation.

Table $1 \mathrm{M}$. interosseus dorsalis manus 1 characteristics in rheumatoid arthritis and osteoarthritis patients

\begin{tabular}{lll}
\hline & Rheumatoid arthritis $(n=5)$ & Osteoarthritis $(n=4)$ \\
\hline MyHC-1 (\%) & $74.4 \pm 15.6$ & $73.3 \pm 19.8$ \\
MyHC-2A (\%) & $23.8 \pm 13.6$ & $23.6 \pm 20.8$ \\
MyHC-2X (\%) & $1.8 \pm 2.3$ & $3.1 \pm 3.2$ \\
CSA MyHC-1 $\left(\mu m^{2}\right)$ & $2534 \pm 714$ & $1906 \pm 773$ \\
CSA MyHC-2 $\left(\mu m^{2}\right)$ & $4263 \pm 1752^{*}$ & $3177 \pm 1201^{*}$ \\
MN MyHC-1 & $2.31 \pm 0.47$ & $1.95 \pm 0.31$ \\
MN MyHC-2 & $3.06 \pm 0.75$ & $2.06 \pm 0.66^{\#}$ \\
MND MyHC-1 $\left(\mu m^{2}\right)$ & $1240 \pm 389$ & $1093 \pm 323$ \\
MND MyHC-2 $\left(\mu m^{2}\right)$ & $1523 \pm 382$ & $1354 \pm 550$ \\
Pax7 $7^{+}$MyHC-1 & $0.038 \pm 0.025$ & $0.020 \pm 0.008$ \\
Pax7 $7^{+}$MyHC-2 & $0.049 \pm 0.075$ & $0.028 \pm 0.025$ \\
CD86 ${ }^{+}$MyHC-1 & $0.036 \pm 0.019$ & $0.034 \pm 0.020$ \\
CD86 $^{+}$MyHC-2 & $0.055 \pm 0.025$ & $0.038 \pm 0.026$
\end{tabular}

Data are shown as mean \pm SD

${ }^{*} P<0.05$, significantly different between MyHC-1 and MyHC-2 fibers within group; ${ }^{\#} P<0.1$, tendency for a between-group difference

CSA cross-sectional area, MN myonuclear number, MND myonuclear domain, MyHC myosin heavy chain 


\section{Abbreviations}

CSA: Cross-sectional area; MyHC: Myosin heavy chain; OA: Osteoarthritis;

RA: Rheumatoid arthritis

\section{Acknowledgements}

Not applicable.

\section{Funding}

Not applicable.

\section{Availability of data and materials}

The datasets of the current study are available from the corresponding author on reasonable request.

\section{Authors' contributions}

SMM, DAg, DAe, and EV collected the data. SMM and MT wrote the paper. $\mathrm{DAe}, \mathrm{EV}$, and MT participated in the conception of the study. SMM, DAg, and MT participated in the analysis of the data. All authors read and approved the final manuscript.

\section{Ethics approval and consent to participate}

Written informed consent was obtained from all participants and the study was conducted according to the bylaws of the institution.

\section{Consent for publication}

Not applicable.

\section{Competing interests}

The authors declare that they have no competing interests.

\section{Publisher's Note}

Springer Nature remains neutral with regard to jurisdictional claims in published maps and institutional affiliations.

\section{Author details}

'Department of Neurology, University Hospital Zurich, Zurich, Switzerland. ${ }^{2}$ Institute of Human Movement Sciences, ETH Zurich, Zurich, Switzerland.

${ }^{3}$ Department of Rheumatology and Clinical Immunology/Allergology, University Hospital Bern, Bern, Switzerland. ${ }^{4}$ Department of Plastic,

Reconstructive and Hand Surgery, University Hospital Bern and University of Bern, Bern, Switzerland. ${ }^{5}$ Research and Performance Centre for Elite Athleticism, OYM, Lorzenparkstrasse 12, 6330 Cham, Switzerland. ${ }^{6}$ Present address: Kieser Training, Zurich, Switzerland.

Received: 26 February 2018 Accepted: 5 March 2018

Published online: 22 March 2018

\section{References}

1. Duijnisveld BJ, Bigot A, Beenakker KG, Portilho DM, Raz V, van der Heide HJ, Visser CP, Chaouch S, Mamchaoui K, Westendorp RG, Mouly V, ButlerBrowne GS, Nelissen RG, Maier AB. Regenerative potential of human muscle stem cells in chronic inflammation. Arthritis Res Ther. 2011;13:R207.

2. Touno M, Senda M, Nakago K, Yokoyama Y, Inoue H. Muscle fiber changes of the vastus medialis in rheumatoid patients. Acta Med Okayama. 1996;50: 157-64.

3. Beenakker KG, Duijnisveld BJ, Van der Linden HM, Visser CP, Westendorp RG, Butler-Brown G, Nelissen RG, Maier AB. Muscle characteristics in patients with chronic systemic inflammation. Muscle Nerve. 2012;46:204-9. 